\title{
Vocalização de anúncio e ampliação da distribuição de Pristimantis crepitans (Bokermann) (Amphibia, Anura, Strabomantidae)
}

\author{
Christine Strüssmann ${ }^{1}$, André Pansonato ${ }^{2} \&$ Davi Soares ${ }^{3}$
}

1. Departamento de Ciências Básicas e Produção Animal, Faculdade de Agronomia, Medicina Veterinária e Zootecnia, Universidade Federal de Mato Grosso (UFMT), Av. Fernando Correa da
Costa, 2367, Boa Esperança, 78060-900, Cuiabá, MT, Brasil. (autor correspondente, christine@ufmt.br)
2. Programa de Pós-Graduação em Ecologia e Conservação da Biodiversidade, Instituto de Biociências, UFMT. (andreufmt@gmail.com)
3. Programa de Pós-Graduação em Biologia Animal, Universidade Federal Rural do Rio de Janeiro, Instituto de Biologia, Rodovia BR 465, Km 7, 23890-000, Seropédica, Rio Janeiro, Brasil.

ABSTRACT. Advertisement call and range extension of Pristimantis crepitans (Bokermann) (Amphibia, Anura, Strabomantidae). New material of Pristimantis crepitans (Bokermann, 1965) was obtained from rocky outcrops located in southwestern borders of the Cerrado biome from central Brazil, and in the western border of the Pantanal wetlands, in the boundaries between Brazil and Bolivia. Individuals were collected and recorded in the type-locality and in four additional municipalities, in two mid-western Brazilian states: Mato Grosso and Mato Grosso do Sul. Besides range extension and morphometric data additional to those in the original description, we here provide a description of the topotypical vocalization of $P$. crepitans, as well as photographs of live specimens and of some of their habitats, for the first time in the literature.

KEYWORDS. Vocalization, geographic distribution, habitat description, rocky outcrops.

RESUMO. Espécimes de Pristimantis crepitans (Bokermann, 1965) foram obtidos em áreas de afloramentos rochosos situadas na borda sudoeste do Cerrado e na borda oeste do Pantanal, próximo à fronteira entre o Brasil e a Bolívia. Indivíduos foram coletados e gravados na localidadetipo e em outros quatro municípios, nos estados de Mato Grosso e Mato Grosso do Sul. Além da ampliação da área de ocorrência e de dados morfométricos adicionais aos da série-tipo, apresentamos pela primeira vez na literatura parâmetros da vocalização topotípica de $P$. crepitans, bem como fotografias de espécimes em vida e de alguns dos hábitats ocupados pelos mesmos.

PALAVRAS-CHAVE. Canto de anúncio, distribuição geográfica, habitat, afloramentos rochosos.

Pristimantis crepitans (Bokermann, 1965) foi descrita com base em apenas três exemplares obtidos

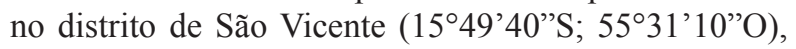
município de Cuiabá, zona rural serrana (cerca de $800 \mathrm{~m}$ de altitude) no sul do estado de Mato Grosso (BoKermann, 1965). A localidade-tipo fica próxima à borda sudoeste do Cerrado, em seus limites com a porção norte da planície inundável do Pantanal.

A vocalização da espécie foi brevemente mencionada na descrição original (BoKERMANN, 1965), mas jamais foi figurada ou teve seus parâmetros espectrais e temporais caracterizados. Embora adequadamente descritos, detalhes da morfologia da parte ventral das mãos e dos pés também não foram ilustrados e apenas um desenho em vista dorsal do holótipo foi fornecido na descrição original (BoKERMANN, 1965). Nenhum registro adicional de $P$. crepitans foi publicado desde sua descrição, o que restringe a caracterização morfológica da espécie à reduzida série-tipo, e sua área de ocorrência, à localidade-tipo.

Sem haver examinado material de $P$. crepitans, Lynch (1980) considerou-a sinônimo de $P$. fenestratus (Steindachner, 1864), anuro ocorrente em formações florestadas e com distribuição amazônica (HEYER \& MuÑoz, 1999). Heyer \& MuÑoz (1999) revalidaram a espécie, após comparar o material-tipo a espécimes de P. fenestratus obtidos em localidades do norte de Mato Grosso. A distinção entre ambas espécies é plenamente corroborada por estudos citogenéticos recentes: $P$. crepitans apresenta número diplóide igual a 22 cromossomos e $P$. fenestratus, 34 (Siqueira et al., 2008; 2009).

Asistemática das espécies de anuros que originalmente estavam incluídas em "Eleutherodactylinae" (sensu Frost, 2010) sofreu profundas alterações após trabalhos moleculares recentes (Frost et al., 2006; Heinicke et al., 2007; Hedges et al., 2008). Os relacionamentos inter- e intra-genéricos em clados originados a partir de novos arranjos taxonômicos nesse grupo (a exemplo de Terrarana, que contém as 439 espécies atualmente conhecidas de Pristimantis; Frost, 2011), entretanto, ainda são escassamente definidos.

Atualmente, Pristimantis crepitans encontra-se alocada no grupo de P. conspicillatus (PADIAL \& DE LA RIVA, 2009). Entretanto, divergências citogenéticas com as demais espécies de Pristimantis já analisadas (SiqueIra et al., 2008; 2009), a singular ocorrência no bioma Cerrado e análises preliminares da ultra-estrutura dos espermatozóides (S. Siqueira, dados inéditos), indicam a possibilidade de uma alocação genérica distinta para P. crepitans (Siqueira et al., 2009).

Com o objetivo de fornecer subsídios que permitam esclarecer a posição sistemática de $P$. crepitans, apresentamos descrição da vocalização topotípica da espécie, dados sobre a morfometria de espécimes adicionais àqueles referidos na literatura e novas localidades de ocorrência nos estados de Mato Grosso e Mato Grosso do Sul.

\section{MATERIAL E MÉTODOS}

Área de estudo. As observações aqui relatadas foram realizadas em municípios do centro-sul e sudoeste de Mato Grosso (Chapada dos Guimarães, Porto Estrela e Rosário Oeste), e extremo noroeste de Mato Grosso do Sul (Corumbá). As localidades em que P. crepitans foi 
registrada até o momento situam-se entre $14^{\circ}-18^{\circ} \mathrm{S}$ e $55^{\circ}$ - $58^{\circ} \mathrm{O}$, em áreas de serras ou planaltos. Em Mato Grosso, essas localidades estão nos limites entre o bioma Cerrado e a borda norte da planície inundável do Pantanal; em Mato Grosso do Sul, na borda oeste do Pantanal, próximo à fronteira entre o Brasil e a Bolívia (Fig. 1).

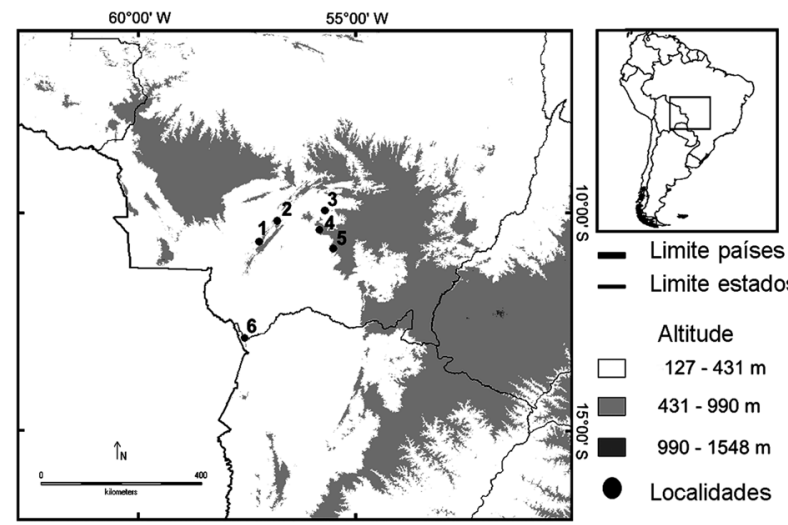

Fig. 1. Ocorrência de Pristimantis crepitans (Bokermann, 1965) nos estados de Mato Grosso (MT) e Mato Grosso do Sul (MS): 1, Porto Estrela, MT; 2, Rosário Oeste, MT; 3, Chapada dos Guimarães (Mata Fria), MT; 4, Chapada dos Guimarães (Serra da Esperança, APM Manso), MT; 5, Santo Antônio do Leverger (Distrito de São Vicente), MT 6, Corumbá, MS

O clima em todas as localidades onde $P$. crepitans foi registrada é do tipo tropical quente e semi-úmido, com duas estações definidas: inverno seco, entre maio e setembro, e verão chuvoso, entre outubro e abril/maio.

$\mathrm{NaChapadados} \mathrm{Guimarães,} \mathrm{a} \mathrm{espécie} \mathrm{foiregistrada,}$ inicialmente, em áreas sob influência do APM Manso (Aproveitamento Múltiplo de Manso), empreendimento hidrelétrico que inundou aproximadamente $428 \mathrm{~km}^{2}$ de fitofisionomias de cerrado, a partir de dezembro de 1999. A maior parte das observações foi realizada na Serra da Esperança, situada no interflúvio entre a margem esquerda do rio Manso e seu principal afluente nessa margem, o rio Casca. A Serra da Esperança integra uma cadeia de pequenas serras isoladas, com altitude em torno de $600 \mathrm{~m}$, natureza arenítica e mesma origem geológica da Chapada dos Guimarães (BRAsIL, 1982).

A temperatura média na região de Manso está em torno de $26^{\circ} \mathrm{C}$ e a média do mês mais frio é superior a $18^{\circ} \mathrm{C}$. A umidade relativa média mensal varia de 60 a $83 \%$, sendo frequente, entre julho e agosto, o registro de valores inferiores a 30\% (AlHo, 2000). A precipitação total anual pode atingir valores superiores a $1.800 \mathrm{~mm}$ em algumas regiões do município de Chapada dos Guimarães (BRASIL, 1982).

No município de Porto Estrela, as médias anuais de temperatura oscilam entre $23^{\circ} \mathrm{C}$ e $25^{\circ} \mathrm{C}$ e a média das máximas fica entre $30^{\circ} \mathrm{C}$ e $32^{\circ} \mathrm{C}$. As precipitações pluviométricas giram em torno de 1300 a $2000 \mathrm{~mm}$ anuais, regime tipicamente amazônico. Níveis mais baixos de precipitação ocorrem no período de maio a setembro e os mais elevados, de dezembro a fevereiro (BRAsil, 1982).

Na Serra do Amolar, extremo noroeste de Corumbá, a temperatura média anual é de $24^{\circ} \mathrm{C}$, a média máxima $34^{\circ} \mathrm{C}$ (entre setembro e outubro) e a média mínima $20^{\circ} \mathrm{C}$ (entre junho e julho). A média anual de pluviosidade é inferior a $1250 \mathrm{~mm}$, sendo mais chuvosos os meses de novembro, dezembro e janeiro, quando chove cerca de 45 a 55\% do total anual (BrAsil, 1982). A elevação máxima aproximada é de $900 \mathrm{~m}$ e a região apresenta solos litólicos distróficos, recobertos por formações vegetais do tipo Contato Savana - Floresta Estacional (BRAsiL, 1982).

Registro e análise das vocalizações. Gravações do canto de anúncio de $P$. crepitans foram obtidas na localidade-tipo (distrito de São Vicente, Santo Antônio do Leverger, Mato Grosso), em duas ocasiões distintas, e em outras três localidades de ocorrência da espécie. Para os registros bioacústicos foram utilizados gravador analógico Sony TCM 5000-EV e microfone dinâmico cardióide Leson DC-44AB ou gravador digital Marantz PMD-660 e microfone direcional Yoga EM-9600.

Digitalização e edição das vocalizações foram realizadas por meio dos aplicativos Cool Edit 96 e Raven Pro 1.3, em frequência amostral de $44,1 \mathrm{kHz}$ e resolução de 16 bits e 256 FFT e, quando necessário, 1024 FFT. A terminologia usada para descrever as vocalizações seguiu Heyer et al. (1990) e Martins \& JiM (2003). Os parâmetros das vocalizações analisadas foram: duração do canto, número de notas por canto, duração de cada nota, taxa de repetição de notas, padrão e número de pulsos, frequências fundamental e dominante.

Gravações-testemunho foram incorporadas ao banco de registros acústicos no Laboratório de Herpetologia do Instituto de Biociências da Universidade Federal de Mato Grosso (IB-UFMT, Cuiabá, Mato Grosso). Os exemplares de P. crepitans associados a estas gravações foram depositados na Coleção Zoológica de Vertebrados da mesma instituição, sob os números de tombo UFMT 1381, 1386 e 10058.

\section{RESULTADOS}

Localidade de ocorrência e hábitats. Na localidadetipo (distrito de São Vicente, Mato Grosso), indivíduos de Pristimantis crepitans foram encontrados em cerrado arbustivo-arbóreo (ou savana arborizada sensu SILVEIRA \& Borges, 2009), com grande quantidade de afloramentos graníticos cujo diâmetro é variável, podendo ser superior, em muitos casos, a $3 \mathrm{~m}$ de diâmetro. À época da descrição original, o distrito de São Vicente pertencia ao município de Cuiabá. Atualmente, em decorrência de subsequentes modificações das fronteiras políticoadministrativas dos municípios do estado de Mato Grosso, a localidade-tipo de $P$. crepitans encontra-se inserida nos limites do município de Santo Antônio do Leverger. A espécie também foi registrada em outros três municípios em Mato Grosso: Chapada dos Guimarães, Porto Estrela e Rosário Oeste.

No município de Chapada dos Guimarães, no planalto de mesmo nome, centro-sul de Mato Grosso, $P$. crepitans foi inicialmente registrada na região do APM Manso. Durante os estudos de impacto ambiental na 
região, realizados entre agosto 1998 e março de 2002, indivíduos da espécie foram encontrados em ambas as margens do rio Casca, a cerca de $100 \mathrm{~km}$ a noroeste da localidade-tipo.

$\mathrm{Na}$ margem direita do rio, em áreas da Fazenda

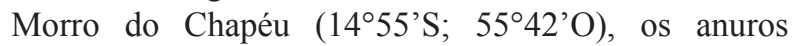
ocupavam ambientes de cerrado stricto sensu, com grande quantidade de afloramentos rochosos (com diâmetro por vezes superior a $1 \mathrm{~m}$ ), que recobrem as encostas e a porção superior da Serra da Esperança. Na margem esquerda do rio Casca, indivíduos de $P$. crepitans foram encontrados em áreas de transição entre florestas de galeria não inundáveis e campo sujo de cerrado, sendo os afloramentos areníticos existentes no local mais esparsos e menores em relação àqueles na Serra da Esperança.

Posteriormente, P. crepitans foi registrada no interior do Parque Nacional da Chapada dos Guimarães, durante estudos visando obter subsídios para o Plano de Manejo dessa unidade de conservação. Em outubro de 2005, no início da estação chuvosa, inúmeros machos adultos em atividade de vocalização foram registrados nos sítios conhecidos como Mata-Fria $\left(15^{\circ} 23^{\prime} \mathrm{S}\right.$;

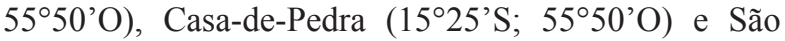
Jerônimo ( $\left(15^{\circ} 25^{\prime} \mathrm{S} ; 5^{\circ} 51^{\prime} \mathrm{O}\right)$.

Pristimantis crepitans também foi registrada em localidades situadas ao longo do conjunto de serras com cristas aproximadamente paralelas conhecido como Província Serrana de Mato Grosso, no sudoeste do estado. Indivíduos foram avistados no interior da Estação Ecológica Serra das Araras (15³9'S; 57¹3’O), no município de Porto Estrela, e também na Fazenda União, próximo às margens do rio Currupira $\left(15^{\circ} 10^{\prime} \mathrm{S}\right.$; $\left.56^{\circ} 46^{\prime} \mathrm{O}\right)$, no município de Rosário Oeste. Em ambas as localidades, os indivíduos de $P$. crepitans ocupavam tanto cerrado stricto sensu como florestas ciliares e florestas semideciduais de encosta, entre 293 e 897 m de altitude.

Em Mato Grosso do Sul, observações, coletas e gravações de $P$. crepitans foram realizadas no interior da Reserva Particular do Patrimônio Natural Acurizal

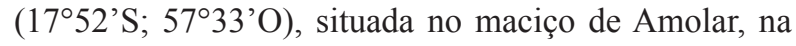
borda oeste do Pantanal.

Em todos os ambientes onde a presença de $P$. crepitans foi registrada, é conspícua a presença de afloramentos rochosos, esparsos e de tamanho variável, de natureza granítica, calcária ou arenítica (Figs 2-4).
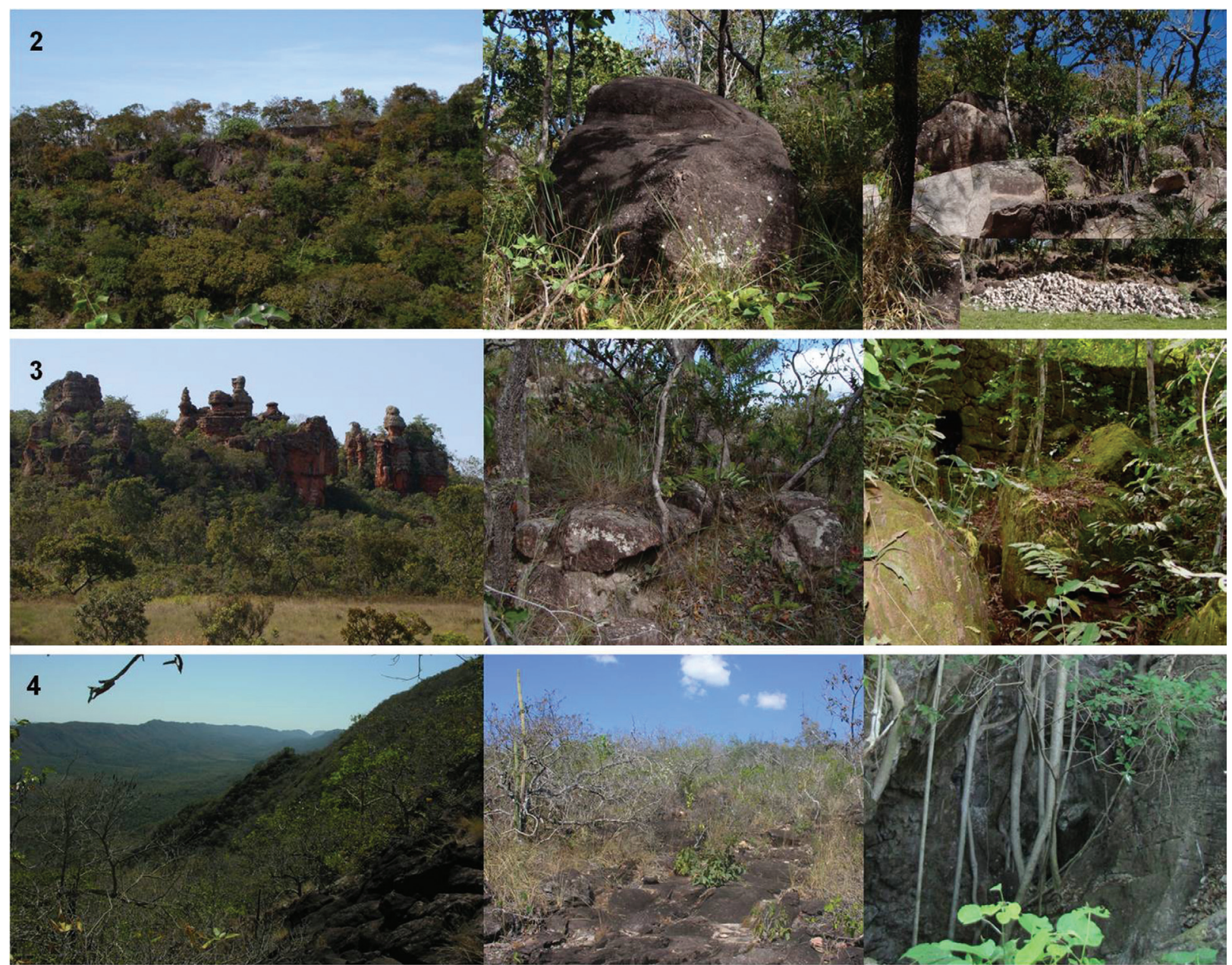

Figs. 2-4. Ambientes onde foram registrados indivíduos de Pristimantis crepitans (Bokermann, 1965) em Mato Grosso, com afloramentos rochosos de natureza: 2, granítica, Serra de São Vicente, Santo Antonio do Leverger; 3, arenítica, Chapada dos Guimarães ou 4, calcária, Porto Estrela e Rosário Oeste. 
Morfologia dos espécimes examinados. As medidas de três topótipos de $P$. crepitans e de outros 29 indivíduos oriundos dos demais municípios são apresentadas na Tab. I. Como subsídios a uma melhor caracterização da espécie, são apresentados detalhes da morfologia do dorso (Fig. 5), ventre (Fig. 6) e cabeça (Fig. 7) e das extremidades (Figs 8-9) de um dos exemplares e ainda o aspecto, em vida, de alguns dos espécimes obtidos em campo (Figs 10-12).

Vocalização. Na serra granítica de São Vicente, Santo Antônio do Leverger, os indivíduos vocalizavam sobre a superfície do solo, junto à base de blocos de granito, no interior de manchas de savana arborizada.
Gravações foram feitas nos fundos de um posto de combustível (1549'08'S; 55³1'25”O), no Km 341 da rodovia BR-364 (antiga BR-29, como mencionado em BoKermann, 1965), em 11 de dezembro de 2001, às 19:50 h, com temperatura do ar a $25^{\circ} \mathrm{C}$.

Gravações adicionais na localidade-tipo foram realizadas nas proximidades do Hotel Pousada São Vicente (15'49'40'S; 55 31'10'O), em 26 de novembro de 2009 , às $21: 20 \mathrm{~h}$, com temperatura do ar a $24^{\circ} \mathrm{C}$. No mesmo hábitat e ocasião, indivíduos de Leptodactylus syphax Bokermann, 1969 e Ameerega braccata (Steindachner, 1864) também foram avistados ou ouvidos com frequência.

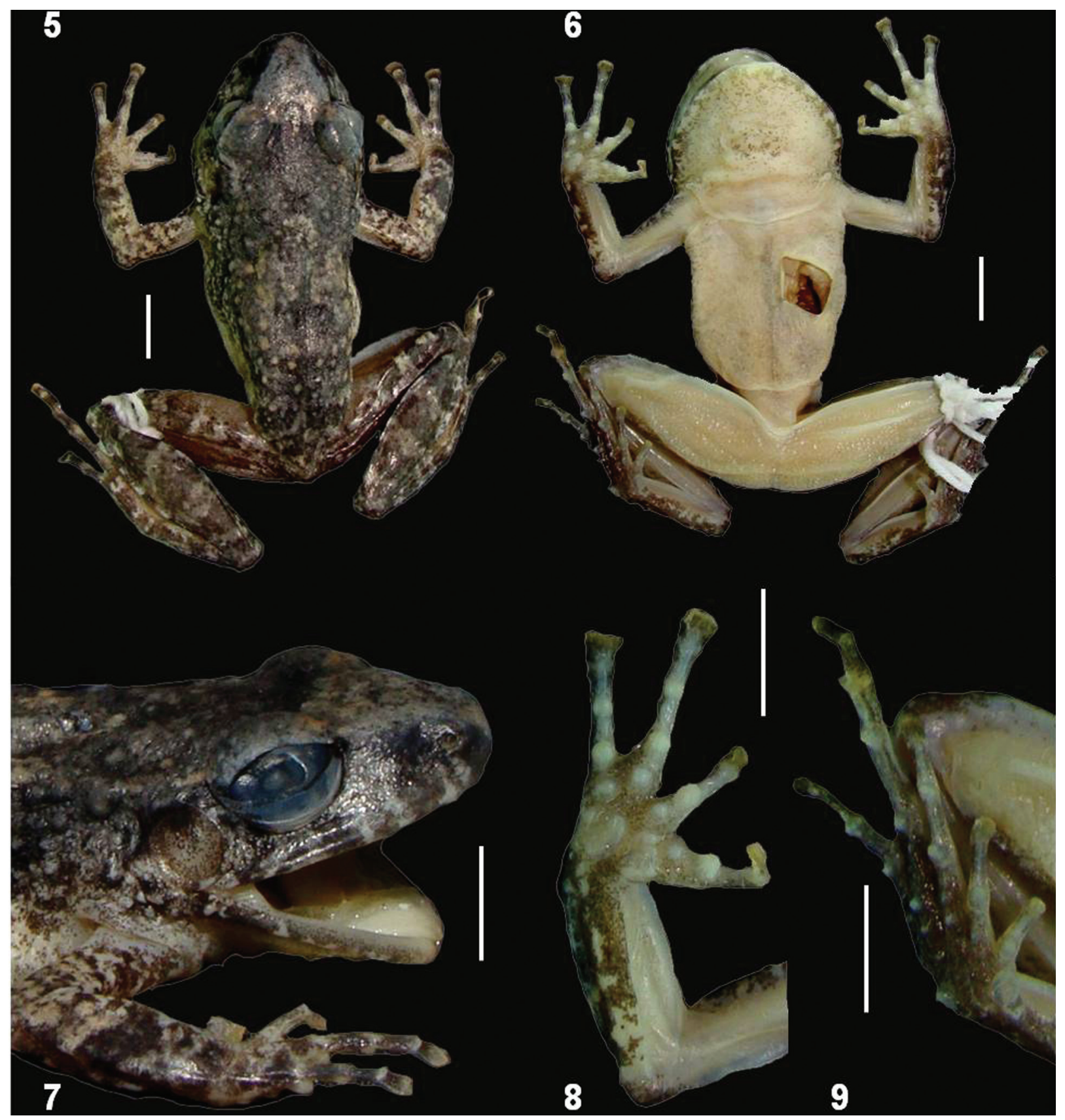

Figs. 5-9. Pristimantis crepitans (Bokermann, 1965) (UFMT 10058): 5, dorso; 6, ventre; 7, cabeça, em perfil; 8, mão, face palmar esquerda; 9 , pé, face plantar esquerda. Escalas $=1 \mathrm{~mm}$. 


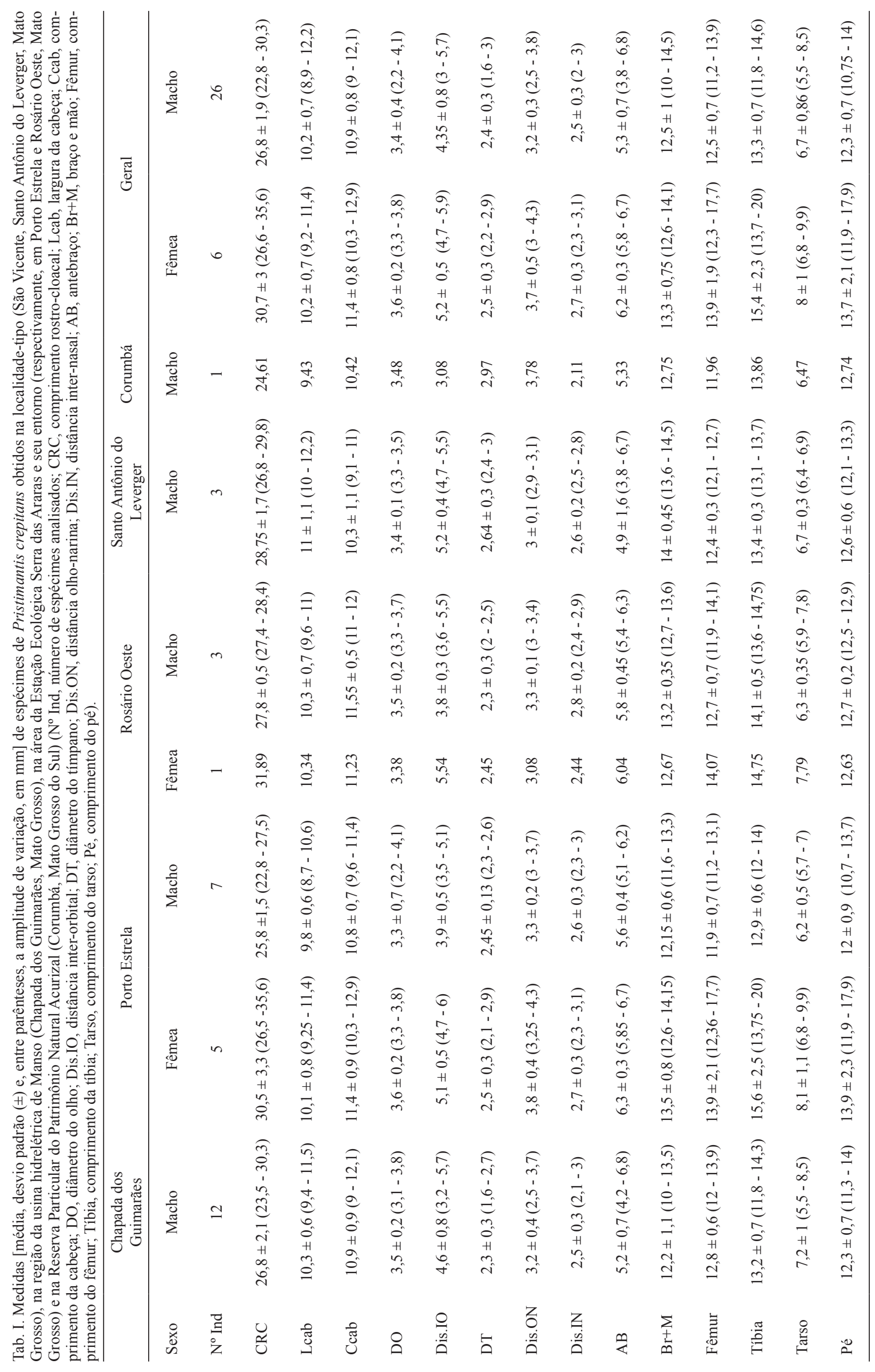




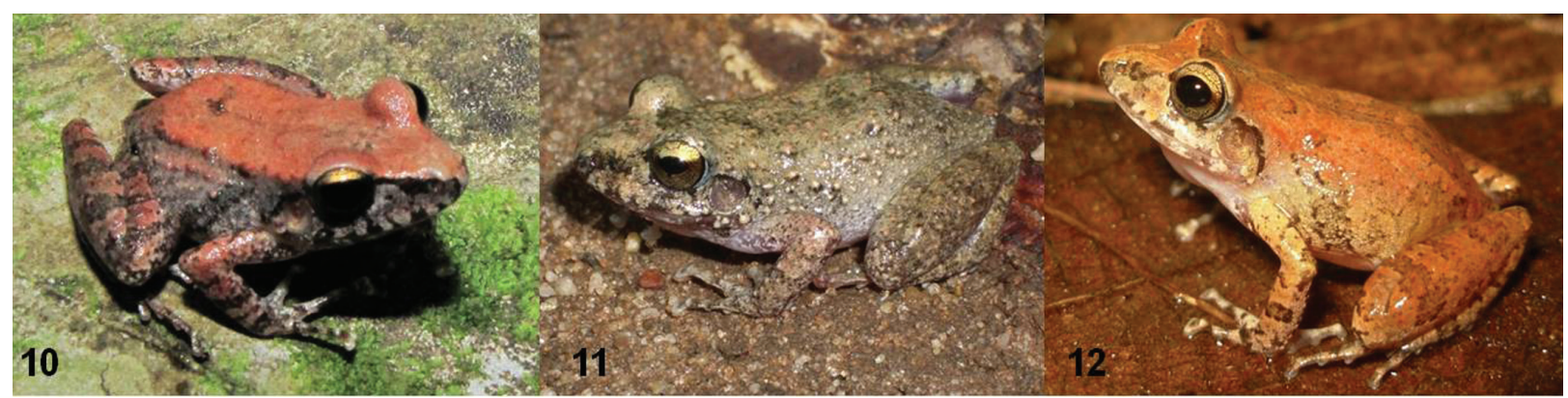

Figs. 10-12. Aspecto em vida de indivíduos adultos de Pristimantis crepitans (Bokermann, 1965), oriundos dos municípios de Porto Estrela (1011) e Chapada dos Guimarães (12), Mato Grosso.

Na região de Manso, em Chapada dos Guimarães, gravações foram realizadas na Serra da Esperança, em áreas da Fazenda Morro do Chapéu (145'이's; $\left.55^{\circ} 42^{\prime} 00^{\prime \prime} \mathrm{O}\right)$, em 25 de novembro de 2000, às 22:15 $\mathrm{h}$, com temperatura do ar a $25^{\circ} \mathrm{C}$. O indivíduo gravado estava vocalizando a cerca de $1,3 \mathrm{~m}$ de altura, em uma pequena reentrância na superfície de um bloco de arenito com cerca de $1,5 \mathrm{~m}$ de diâmetro, em local aberto, em meio ao cerrado arbustivo-arbóreo. Muitos outros indivíduos foram encontrados no mesmo local, em situação semelhante, com tempo chuvoso.

Na Estação Ecológica de Serra das Araras, Porto Estrela, os indivíduos vocalizavam junto ao solo, sobre a serapilheira, em floresta estacional semidecidual com predominância de babaçu Orbygnia speciosa. As gravações foram realizadas em floresta estacional semidecidual na margem direita do córrego Camarinha ( $15^{\circ} 29^{\prime} 08^{\prime \prime} \mathrm{S}$; $\left.57^{\circ} 05^{\prime} 52^{\prime \prime} \mathrm{O}\right)$, em 17 e 18 de dezembro de 2009 , às 22:30 e 00:20 h, com temperatura do ar a $24^{\circ} \mathrm{C}$, respectivamente. Nesta mesma localidade também foram avistados indivíduos vocalizando sobre afloramentos calcários ou areníticos, de forma semelhante ao relatado para a região do APM Manso.

Em outubro de 2009, início do período chuvoso no Brasil Central, vários indivíduos de $P$. crepitans foram coletados durante expedição a áreas no entorno da Estação Ecológica Serra das Araras, nas proximidades do córrego Currupira, em Rosário Oeste. Embora todas as fêmeas capturadas estivessem com complemento ovariano perceptível, aparentemente prontas para desovar, nenhuma vocalização foi ouvida.

$\mathrm{Na}$ Reserva Particular do Patrimônio Natural Acurizal, região da Serra do Amolar, o indivíduo gravado vocalizava em uma área de abrupta transição entre a mata ciliar e um encrave de cerrado stricto sensu. A gravação foi obtida em 29 de outubro de 2003, às 22:00 h, com temperatura do ar $26^{\circ} \mathrm{C}$, nas proximidades da margem esquerda do córrego Fundão (17 $52^{\circ} 39^{\prime \prime}$; $57^{\circ} 33^{\prime} 28^{\prime \prime}$ ), afluente da margem direita do rio Paraguai. Pristimantis crepitans possui canto simples com duração de $0,04 \mathrm{~s}$, intervalo de $1,8 \mathrm{~s}$ (Tab. II), podendo emitir em média 47 cantos/minuto ( $14-71$ cantos/minuto). $\mathrm{O}$ canto é formado por duas notas distintas, com duração de $0,013 \mathrm{~s}$ e com intervalo entre si de $0,015 \mathrm{~s}$. O canto apresenta faixa de frequência entre $1,4-4,6 \mathrm{kHz}$ e frequência dominante média de $2,67 \mathrm{kHz}$. A frequência dominante média da primeira nota é $2,7 \mathrm{kHz}$, e da segunda, 2,8 kHz (Figs 13-15). A primeira nota pode ser repetida de forma sequenciada, o que somente foi registrado para o indivíduo gravado em São Vicente, no início do turno de vocalização, quando poucos indivíduos estavam vocalizando.

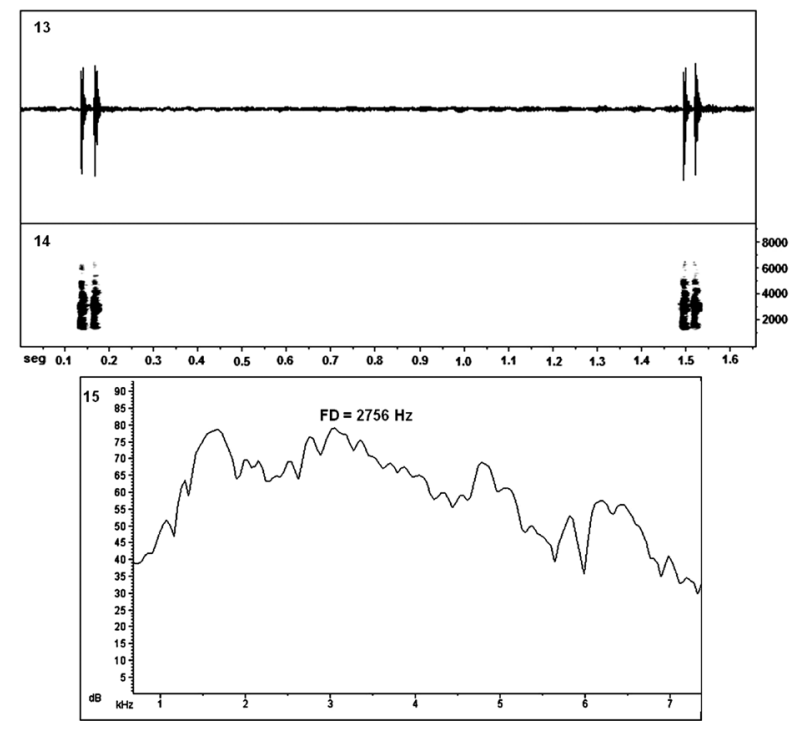

Figs. 13-15. Oscilograma (13), sonograma (14) e espectro de potência (15) de dois cantos de anúncio de Pristimantis crepitans (Bokermann, 1965) (UFMT 10058). Gravado em 26.11.2009, 21:20 h, $24^{\circ} \mathrm{C}$, nas proximidades do Hotel Pousada São Vicente (15 $45^{\circ} 40^{\prime}$ 'S; 55³1'10”O), distrito de São Vicente, Santo Antônio do Leverger, Mato Grosso, Brasil.

\section{DISCUSSÃO E CONCLUSÃO}

A presença de $P$. crepitans em localidades com abundância de afloramentos de natureza arenítica (Fig. 3), como na Chapada dos Guimarães, ou calcária, como em Porto Estrela e Rosário Oeste (Fig. 4), evidencia que a espécie não é endêmica da formação granítica existente na Serra de São Vicente, como anteriormente sugerido (HeYER \& MuÑOZ, 1999).

Se por um lado os novos registros contribuem para melhor compreensão do status de conservação da espécie, considerada por HeYer et al. (2004) como deficiente de dados, por outro as novas observações na localidade-tipo de $P$. crepitans são motivo de preocupação. Em São Vicente, indivíduos foram 
Tab. II. Parâmetros bioacústicos de machos de Pristimantis crepitans gravados em Santo Antônio do Leverger (distrito de São Vicente) e Porto Estrela (Estação Ecológica Serra das Araras), Mato Grosso, Brasil. Os valores apresentados referem-se à média dos cantos, desvio padrão ( \pm ) e, entre parênteses, a amplitude de variação.

\begin{tabular}{|c|c|c|c|}
\hline & São Vicente & Porto Estrela & Média \\
\hline $\mathrm{n}^{\circ}$ indivíduos gravados & 1 & 2 & \\
\hline $\mathrm{n}^{\mathrm{o}}$ cantos analisados & 31 & 12 & \\
\hline Duração do canto (s) & $0,04 \pm 0,004(0,03-0,05)$ & $0,04 \pm 0,004(0,04-0,05)$ & $0,04 \pm 0,004(0,03-0,05)$ \\
\hline Intervalo entre cantos (s) & $1,14 \pm 0,3(0,7-1,9)$ & $3,7 \pm 2,7(1,2-11,5)$ & $1,85 \pm 1,8(0,7-11,5)$ \\
\hline Duração da $1^{\mathrm{a}}$ nota (s) & $0,011 \pm 0,001(0,006-0,01)$ & $0,012 \pm 0,003(0,007-0,02)$ & $0,012 \pm 0,002(0,006-0,02)$ \\
\hline Duração da $2^{\mathrm{a}}$ nota (s) & $0,012 \pm 0,002(0,007-0,01)$ & $0,014 \pm 0,004(0,008-0,02)$ & $0,013 \pm 0,003(0,007-0,02)$ \\
\hline Intervalo entre notas (s) & $0,015 \pm 0,002(0,01-0,02)$ & $0,015 \pm 0,001(0,01-0,02)$ & $0,015 \pm 0,002(0,01-0,02)$ \\
\hline $\begin{array}{l}\text { Frequência dominante do canto } \\
(\mathrm{Hz})\end{array}$ & $2778,7 \pm 290(2067,2-2856,3)$ & $2560,1 \pm 347(2067,2-3100,8)$ & $2677,3 \pm 333(2067,2-3100,8)$ \\
\hline $\begin{array}{l}\text { Frequência dominante da } 1^{\text {a }} \\
\text { nota }(\mathrm{Hz})\end{array}$ & $2786,3 \pm 145,9(2348,2-2966,3)$ & $2609,1 \pm 350,6(2203,7-3174)$ & $2736,8 \pm 232,1(2203,7-3174)$ \\
\hline $\begin{array}{l}\text { Frequência dominante da } 2^{\mathrm{a}} \\
\text { Nota }(\mathrm{Hz})\end{array}$ & $2819,9 \pm 151(2345,0-2993,7)$ & $2860,6 \pm 272,3(2519,1-3313,8)$ & $2831,3 \pm 189,9(2345,0-3313,8)$ \\
\hline
\end{tabular}

encontrados em situação semelhante àquela referida por BOKERMANN (1965) na descrição original, há mais de 40 anos: vocalizando no solo, em encostas de morros, entre blocos de granito existentes em meio ao cerrado do tipo savana arbórea aberta (BRASIL, 1982). Entretanto, as jazidas graníticas em São Vicente vêm sendo exploradas há décadas e os níveis e padrões atuais de exploração (Fig. 2) são alarmantes. Inicialmente localizada e limitada a uma ou poucas pedreiras, a exploração atualmente vem sendo feita por trabalhadores isolados, os quais cortam artesanalmente os grandes blocos graníticos, simultaneamente, em diversos locais em meio ao cerrado, havendo múltiplos e esparsos pontos de perturbação das condições naturais.

As principais diferenças morfométricas entre exemplares da série-tipo de $P$. crepitans medidos por BoKERMANN (1965) e os espécimes adicionais aqui caracterizados referem-se ao diâmetro dos olhos, do tímpano, e à distância entre o olho e a narina. Os valores destes atributos são consistentemente maiores no holótipo e no parátipo do que em qualquer um dos topótipos ou espécimes obtidos em Chapada dos Guimarães, Porto Estrela, Rosário Oeste e Corumbá. Estes resultados assemelham-se àqueles obtidos por HEYER \& MuÑoz (1999), que também obtiveram medidas de comprimento rostro-cloacal inferiores àquelas referidas na descrição original, ao re-examinarem os tipos.

A ausência de dimorfismo sexual relatada por Heyer \& MuÑoz (1999) e reafirmada por PAdial \& DE LA Riva (2005), com base no escasso material-tipo, não foi corroborada pelo exame do material adicional aqui referido. Machos e fêmeas de $P$. crepitans apresentam dimorfismo sexual quanto ao comprimento rostrocloacal (CRC), sendo as fêmeas $(n=6)$ significativamente maiores ( $p=0,023 ; t=-2,361$, ajustamento de Bonferroni) que os machos $(n=26)$.

As vocalizações de indivíduos de $P$. crepitans registradas na localidade-tipo e na Serra das Araras, em Porto Estrela, apresentam grande semelhança, particularmente quanto à duração do canto e das notas, bem como do intervalo entre notas. Já o intervalo entre os cantos varia muito entre os exemplares gravados, o que parece estar relacionado ao início do turno de vocalização. Também há diferenças sutis na frequência dominante dos cantos obtidos nas duas localidades, sendo essa frequência um pouco mais elevada no canto do indivíduo gravado na localidade-tipo. Tais diferenças podem corresponder a variações populacionais, sendo necessário analisar maior número de indivíduos.

A vocalização de $P$. crepitans foi originalmente descrita como "duas notas seguidas semelhante a um estalido curto e áspero que são repetidas com frequência" (BoKermanN, 1965). A produção de sons semelhantes a estalidos também foi relatada para Ischnocnema juipoca Sazima \& Cardoso, 1978 (Brachycephalidae), espécie com ampla ocorrência no sudeste brasileiro (HADDAD et al., 1988). O canto de I. juipoca é referido como sendo composto por "uma série curta e ascendente de estalidos, semelhantes ao som produzido por castanholas. Cada série consiste em dez notas curtas, de duração semelhante" (SAZIMA \& CARdoso, 1978; HAdDAD et al., 1988).

Outro membro de Terrarana cujos machos parecem também produzir estalidos ao vocalizar é Oreobates cruralis (Boulenger, 1902) (Strabomantidae), de regiões andinas do sul do Peru e oeste da Bolívia. Sua vocalização, segundo MARQUEz et al. (1995a), consiste em uma única nota, multipulsionada, emitida em média 19,7 vezes em 
cada minuto, a intervalos regulares. Contudo, a análise espectral do canto disponível no guia sonoro de anfíbios da Bolívia (DE LA Riva et al., 2002) mostra que o canto de $O$. cruralis é composto, na realidade, por 10-14 notas simples. Assim como I. juipoca, portanto, o canto de $O$. cruralis apresenta número de notas consideravelmente superior ao registrado em $P$. crepitans.

Em Oreobates barituensis Vaira \& Ferrari, 2008, a configuração espectral das notas do canto de anúncio (VAIRA \& FERRARI, 2008) é, aparentemente, semelhante àquela obtida para $P$. crepitans, mas o canto consiste em um trinado composto por 5-6 notas (ao invés de duas, como em $P$. crepitans).

As peculiaridades bioacústicas e de uso de hábitats referidas no presente trabalho, em conjunto com divergências citogenéticas (SIQUEIRA et al., 2008; 2009) e análises da ultraestrutura dos espermatozóides (S. Siqueira, dados inéditos), reforçam a necessidade de revisão da alocação genérica de Pristimantis crepitans.

Agradecimentos. A Jair Serratel, proprietário da Fazenda Morro do Chapéu, e a Cleber J. R. Alho, coordenador do Programa de Monitoramento e Resgate da Fauna do APM Manso, bem como à Eletronorte e Furnas Centrais Elétricas S.A., pelo apoio logístico na região de Manso; a Rafael M. Valadão, pelo apoio na Estação Ecológica Serra das Araras; a Ricardo A. Kawashita-Ribeiro, pela elaboração do mapa; à Fundação ECOTRÓPICA e PROBIO/MMA, pelo apoio durante a realização de inventários da biodiversidade na RPPN Acurizal e a Rogério Bastos (UFG) e Luciano de A. Moura (FZB-RS) por valiosas sugestões ao texto final. $\mathrm{Na}$ fase final de elaboração do artigo contamos com o apoio logístico do Núcleo Interdisciplinar de Estudos Faunísticos (NIEFA; processo FAPEMAT 447441/2009).

\section{REFERÊNCIAS BIBLIOGRÁFICAS}

Almo, C. J. R. 2000. Paisagens e enfoque. In: Alho, C. J. R.; Conceição, P. N.; Constantino, R.; Schlemmermeyer, T.; Strüssmann, C. Vasconcelos, L. A. S.; Oliveira, D. M. M. \& Schneider, M. eds. Fauna silvestre da região do Rio Manso, MT. Brasília, Ministério do Meio Ambiente, IBAMA, Centrais Elétricas do Norte do Brasil. p. 19-29.

Bokermann, W. C. A. 1965. Três novos batráquios da região central de Mato Grosso, Brasil (Amphibia, Salientia). Revista Brasileira de Biologia 25(3):257-264.

Brasil. Ministério das Minas e Energia. 1982. Projeto RADAMBRASIL. Folha SD.21 Cuiabá; geologia, geomorfologia, pedologia, vegetação e uso potencial da terra. Levantamento de Recursos Naturais, v. 26. Rio de Janeiro, Projeto RADAMBRASIL. 544 p.

De la Riva, I.; Reichle, S.; Köhler, J.; Lötters, S.; Bosch, J.; Mayer, S.; Hennessey, A. B. \& Padial, J. M. 2002. Guia Sonora de las Ranas y Sapos de Bolivia. CD 2. In: MÁrquez, R.; De la Riva, I.; Bosh, J. \& Matheu, E. eds. Barcelona, Alosa-Ahe-MNCN
Frost, D. R. 2011. Amphibian Species of the World: an Online Reference. New York, American Museum of Natural History. Disponível em: <http://research.amnh.org/vz/herpetology/ amphibia/>. Acesso em: 23.12.2011.

Frost, D. R.; Grant, T.; Faivovich, J.; Bain, R. H.; Haas, A.; Haddad, C. F. B.; SÁ, R. O. De; Channing, A.; Wilkinson, M.; Donnellan, S. C.; Raxworthy, C. J.; Campbell, J. A.; Blotto B. L.; Moler, P.; Drewes, R. C.; Nussbaum, R. A.; Lynch, J. D.; Green, D. M. \& WheELER, W. C. 2006. The amphibian tree of life. Bulletin of the American Museum of Natural History 297:1-370.

Haddad, C. F. B; Andrade, G. V. \& Cardoso, A. J. 1988. Anfíbios Anuros no Parque Nacional da Serra da Canastra, Estado de Minas Gerais. Brasil Florestal 64:9-20.

Hedges, S. B.; Duellman, W. E. \& Heinicke, M. P. 2008. New World direct-developing frogs (Anura, Terrarana): Molecular phylogeny, classification, biogeography, and conservation. Zootaxa 1737:1-182.

Heinicke, M. P.; Duellman, W. E. \& Hedges, S. B. 2007. Major Caribbean and Central American frog faunas originated by ancient oceanic dispersal. Proceedings of the National Academy of Sciences of the United States of America 104(24):10092-10097.

Heyer, R.; Colli, G. \& Silvano, D. 2004. Eleutherodactylus crepitans. In: IUCN 2010. IUCN Red List of Threatened Species.Version 2010.1. Disponível em: $<$ www.iucnredlist.org $>$. Acesso em: 19.05.2010.

Heyer, W. R. \& Muñoz, A. M. 1999. Validation of Pristimantis crepitans Bokermann, 1965, notes on the types and type locality of Telatrema heterodactylum Miranda-Ribeiro, 1937, and description of a new species of Eleutherodactylus from Mato Grosso, Brazil (Amphibia: Anura: Leptodactylidae). Proceedings of the Biological Society of Washington 112(1):1-18.

Heyer, W. R.; Rand, A. S.; Cruz, C. A. G.; Peixoto, O. L. \& Nelson, C. E. 1990. Frogs of Boracéia. Arquivos de Zoologia 31(4):231-410.

LyNCH, J. D. 1980. A taxonomic and distributional synopsis of the Amazonian frogs of the genus Eleutherodactylus. American Museum Novitates 2696:1-24.

Marquez, R.; De la Riva, I. \& Bosch, J. 1995. Advertisement calls of Bolivian Leptodactylidae (Amphibia, Anura). Journal of Zoology 237:313-336.

Martins, I. A. \& JiM, J. 2003. Bioacoustic analysis of advertisement call in Hyla nana and Hyla sanborni in Botucatu, São Paulo, Brazil. Brazilian Journal of Biology 63(3):507-516.

Padial, J. M. \& De la Riva, I. 2005. Rediscovery, redescription and advertisement call of Eleutherodactylus heterodactylus (Miranda Ribeiro, 1937) (Anura: Leptodactylidae), and notes on other Eleutherodactylus. Journal of Herpetology 39:372-379.

2009. Integrative taxonomy reveals cryptic Amazonian species of Pristimantis (Anura: Strabomantidae). Zoological Journal of the Linnean Society 155:97-122.

Sazima, I. \& Cardoso, A. J. 1978. Uma espécie nova de Eleutherodactylus do Sudeste Brasileiro (Amphibia, Anura, Leptodactylidae). Revista Brasileira de Biologia 38(4):921-925.

Siqueira, S.; Aguiar JR., O.; Strüssmann, C.; Del-Grande, M. L. \& Recco-Pimentel, S. M. 2008. Chromosomal analysis of three Brazilian "eleutherodactyline" frogs (Anura, Terrarana), with suggestion of a new species. Zootaxa 1860:51-59.

Siqueira, S.; Aguiar Jr., O.; Pansonato, A.; Giaretta, A. A.; Strüssmann, C.; Martins, I. \& Recco-Pimentel, S. M. 2009. The karyotype of three Brazilian Terrarana frogs (Amphibia, Anura) with evidence of a new Barycholos species. Genetics and Molecular Biology 32(3):470-476.

VARIA, M. \& Ferrari, L. 2008. A new species of Oreobates (Anura: Strabomantidae) from the Andes of northern Argentina. Zootaxa 1908:41-50.

Recebido em 25 de outubro de 2010. Aceito em 18 de dezembro de 2011. ISSN 0073-4721

Artigo disponível em: www.scielo.br/isz

Impresso e distribuído em 2012. 